\title{
MONTE CARLO SIMULATION OF DIFFUSION ON THE CUBIC PERCOLATION LATTICE ABOVE THE THRESHOLD
}

\author{
O. J. Poole \\ School of Mathematical Studies University of Portsmouth, \\ Portsmouth, PO12EG, England,e-mail: poole@sms.port.ac.uk
}

(Received September 3, 1996)

\begin{abstract}
Recent work on the square percolation lattice resolved the discrepancy that existed between the scaling behaviour of percolation diffusion and the conductivity of the associated random resistor network. This discrepancy is first recalled, then attention is directed to the cubic percolation lattice where diffusion is again shown to scale with the same critical exponent as the lattice conductivity. This is the first time the scaling behaviour of diffusion and conduction on the cubic lattice have been reconciled. Finally, evidence is presented which suggests that the relaxation rate of percolation diffusion has scaling behaviour with a scaling exponent similar in value to that which governs the correlation length of the lattice.
\end{abstract}

Key words: Monte Carlo, percolation, critical behaviour.

PACS number(s): 64.60.A, 02.70.L

\section{INTRODUCTION}

The study of percolation theory first arose from a problem set by the English mathematicians Broadbent and Hammersly [1] who were interested in the flow of a liquid or gas through a disordered material. Broadbent [2] commented that the percolation problem would be ideal for Monte Carlo computer study and such work lead to sophisticated usage of the extremely low power computers of the time. Since inception, percolation theory has evolved into a branch of statistical mechanics in its own right, finding applications as diverse as particle physics, cosmology, semi-conductors, and beyond physics into chemistry and the life sciences [3]. In short, Monte Carlo work has played a large part in developing an understanding of percolation theory [4] and still has an important role.

There are two types of lattice studied in percolation theory, the bond model and the site model. Both lattices have uniform spacing and are of infinite extent. Each model has either the bonds or sites of the lattice occupied with a probability $p$. The critical point of the percolation lattice, $p_{c}$, is defined as the value of $p$ at which an infinite cluster of nearest neighbour occupied bonds or sites spans the lattice; for all $p<p_{c}$ only finite size clusters exist. The value of $p_{c}$ is dependent on the lattice type and dimension, on the square lattice $p_{c}=0.5927 \ldots$ (site) and $p_{c}=1 / 2$ (bond) and on the cubic lattice $p_{c}=0.3116 \ldots$ (site) and $p_{c}=0.2488 \ldots$ (bond) [4]. It is convention that fractional values denote analytical derivation and decimal values denote numerical estimates.

Various cluster properties, such as the proportion of sites belonging to the infinite cluster, $P_{\infty}$, or the correlation length, $\xi$, have a critical dependence on the distance $\left(p-p_{c}\right)[4,5]$. This scaling behaviour is known exactly in the case of the two dimensional bond lattice by analogy to the Potts model $[6,7]$. Universality [8] implies that the values of these exponents depend only on the dimension of the system, lattice structure being irrelevant.

It is ironic that the behaviour of dynamic processes such as diffusion or conduction, which were the inspiration for percolation theory, have so far proven to be less tractable by analysis. Unlike the static cluster properties, dynamic processes have no analogous property found in other statistical mechanical models. Although some progress has been made by theoretical study, work has been carried out by Adler et al [9], Kesten [10] and Golden [11] for example, dynamic processes are studied mainly by Monte Carlo simulation.

Monte Carlo studies of lattice conductivity have produced clear evidence of scaling behaviour [12-17] with broad agreement found between earlier and later work. In contrast, the results of diffusion simulations are inconsistent. The Einstein relation implies that conductivity and diffusivity have the same critical dependence on $\left(p-p_{c}\right)$, governed by the dynamic exponent $\mu$, as discussed in [18]. Diffusion simulations at the threshold $[19,20]$ gave a value of $\mu$ similar to that obtained from conductivity measurements but until recently simulations of diffusion above the threshold did not $[21,22]$. Then, Monte Carlo work [23] on the square site lattice showed that the anticipated scaling behaviour of classical diffusivity could be observed only if the very slow relaxation behaviour of percolation diffusion was accounted for.

In this paper, attention is now turned to the behaviour of percolation diffusion on the cubic site lattice. Simulations of percolation diffusion were carried out above the threshold near the critical point to see whether the same type of relaxation behaviour found in two dimensions is also evident in three thus allowing the correct scaling behaviour to be determined. The remainder of the paper is laid out in the following manner. Section Two gives a brief review of the scaling theory for dynamic processes. Then, Section Three provides some notes on the simula- 
tions carried out here, the results of which are analysed in Section Four. The paper is then concluded in Section Five.

\section{SOME SCALING THEORY FOR DYNAMIC PROCESSES}

Several properties of percolation lattices have been found to scale with the distance $\left(p-p_{c}\right)[4,5,24]$. For example, $P_{\infty}$ scales as $P_{\infty} \propto\left(p-p_{c}\right)^{\beta}$ and $\xi$ as $\xi \propto\left(p-p_{c}\right)^{\nu}$. In $d=2$ dimensions $\beta=5 / 36$ and $\nu=4 / 3$ and in $d=3$ dimensions $\beta=0.41 \ldots$ and $\nu=0.88 \ldots[4]$.

Dynamic processes on percolation lattices are confined to taking place on occupied sites. The conductivity is defined as follows [4]. If a unit voltage is applied across opposite sides of a lattice of edge length $L$, then the conductivity, $\Sigma$, is the current flowing through the lattice multiplied by $L^{2-d}$, where $d$ is the dimensionality of the lattice. When $L=\infty, \Sigma=0$ for all $p<p_{c}$ and above the threshold $\Sigma$ increases to a maximum as $p$ tends towards unity. Although large scale conductivity must occur via the backbone, there is no simple relationship between the behaviour of $\Sigma$ and $P_{\infty}$ as might be expected.

A property related to conduction is diffusion, but the two properties have a subtle difference. Whereas conductivity is time independent, having a dependence only on the structure of the clusters, diffusion is a time dependent property. Diffusion on percolation clusters is modelled by the following simple algorithm, termed the Ant in the Labyrinth [25]. A diffusing particle starting from an occupied site selects a nearest neighbour site at random. If the chosen site is also occupied then the particle moves to it, otherwise the particle remains stationary. These actions constitute a single time step irrespective of whether or not the particle has moved. Progress of the diffusing particle on the lattice is measured by the mean square distance $\left\langle R^{2}\right\rangle$ moved in $N$ steps.

Below the threshold, where there is no infinite cluster, the trajectory of a diffusing particle must ultimately be restricted by the cluster perimeter. This means that the distance $\left\langle R^{2}\right\rangle$ approaches a limiting value and behaves like $\left\langle R^{2}\right\rangle=R_{\infty}^{2}-A \exp \left[-\left(\frac{N}{\tau_{<}}\right)^{w}\right]$ for large $\mathrm{N}$, where $R_{\infty}^{2}$ is the saturation distance, $A$ and $w$ are constants and $\tau_{<}$is the characteristic saturation time $[19,22,26]$. The quantities $R_{\infty}^{2}$ and $\tau_{<}$were conjectured [26] to follow the scaling laws $R_{\infty}^{2} \propto\left(p-p_{c}\right)^{2 \nu-\beta}$ and $\tau_{<} \propto\left(p-p_{c}\right)^{\vartheta}$. Supporting evidence of these scaling laws was provided by Monte Carlo simulation $[19,22,26]$.

Above the threshold, diffusion is unbounded. Gefen et al [27] conjectured that diffusion was initially anomalous on length scales less than $\xi$ and then crossed over to classical behaviour after some characteristic time $\tau_{>}$. In the anomalous regime, diffusion follows the behaviour $\tilde{R}=\left\langle R^{2}\right\rangle \propto N^{2 k} ; k<1 / 2$ but in the classical regime, diffusion behaves according to the well known diffusion law, $\left\langle R^{2}\right\rangle=D N$. At $p=1$, where $\xi=0$, diffusion is classical for all $N$ and $D$ is normalised to unity. For $p_{c}<p<1$ it was presumed by Gefen et al that diffusion would be classical after the crossover point but with $0<D<1$.
At the threshold, where $\xi=\infty$, diffusion is anomalous for all $\mathrm{N}$.

As implied by the Einstein diffusion relation it is expected [13] that $\Sigma \propto D$ which means that both properties share the same critical exponent,

$$
\Sigma \propto D \propto\left(p-p_{c}\right)^{\mu}
$$

There is no general scaling relation valid for all $d$ which expresses the dynamic exponent $\mu$ solely in terms of static exponents such as $\beta$ or $\nu$. One relation was conjectured by Alexander and Orbach [28],

$$
\mu=1 / 2[\nu(3 d-4)-\beta]
$$

which gives $\mu=91 / 72$ when $d=2$ and $\mu \cong 1.995$ when $d=3$. Recent simulations, however, do not support this conjecture in $d=2[20,23]$, although there is evidence to suggest that it may be true for higher dimensions $[4,29]$.

Gefen et al [27] and Pandey et al [19] derived the scaling relation $2 k=\frac{2 \nu-\beta}{2 \nu-\beta+\mu}$ which gives $\mu$ in terms of static exponents and the diffusion exponent $k$. Simulations of diffusion at the threshold gave $k=0.332 \pm 0.002$ and $k=0.20 \pm 0.01$ in $d=2$ and $d=3$ dimensions respectively from which $\mu=1.28 \pm 0.02$ and $\mu=2.0 \pm 0.2$ were obtained, using values of the critical exponents slightly different to those cited here [19]. These agree with $\mu=1.299 \pm 0.002$ and $\mu=2.003 \pm 0.047$ obtained from the latest conductivity measurements $[16,17]$.

Diffusion simulations above the threshold gave values of $\mu$ which were too low, i.e $\mu=0.99 \pm 0.02$ and $\mu=1.72 \pm 0.03$ for the square and cubic lattice respectively [21] and $\mu=1.70 \pm 0.05$ on the cubic lattice [22]. This prompted some [22] to suggest that there were in fact 2 dynamic critical exponents, one for conduction and one for diffusion. It was shown finally [23] that percolation diffusion is classical in the limit of $N \rightarrow \infty$ only. When the relaxation to the classical limit was accounted for, scaling behaviour of $D$ compatible with the Einstein relation was observed with a dynamic exponent $\mu=1.291 \pm 0.024$ in agreement with [16] but not, however, with the Alexander-Orbach conjecture (2).

\section{NOTES ON THE SIMULATION}

Percolation diffusion modelled by the Ant in the Labyrinth Algorithm (AIL) [25] is ideal for Monte Carlo simulation where a large number of diffusing particles perform an ensemble of random walks on the lattice. The behaviour of $\left\langle R^{2}\right\rangle$ with $N$ is determined for a specific probability $p$ in the following manner. Each walk starts from an occupied site, the origin. Then it takes $N$ steps according to the rules of percolation diffusion defined above. After each step or intervals of steps the position of the ant relative to the origin is noted and the distance $R^{2}$ evaluated. If a large number of walks are executed on a large number of lattice configurations then the mean square distance $\left\langle R^{2}\right\rangle$ can be evaluated for 
values of $N$. With the advent of fast desk top computers, AIL simulations can now be carried out in relatively little time.

The new results reported in this paper were carried out on an Intel 486/66 based computer. The biggest constraint on the simulation was the amount of memory available. The size of the lattice was therefore limited in this instance to an edge length of $L=100$. The simulations were carried out for the range $0.33 \leq p \leq 0.39$. For each probability $p, 200$ walks were carried out on each of 100 different lattice configurations giving $2 \times 10^{4}$ walks in total. Periodic boundary conditions were imposed on the lattice and the distance $R^{2}$ was evaluated for every hundredth step. The simulation for each probability $p$ took approximately 36 hours.

\section{RESULTS AND DISCUSSION}

Results from the simulations were obtained in the form $\left\langle R^{2}\right\rangle$ vs. $N$ and looked typically like that shown in figure 1 which shows the graph for $p=0.34$. The behaviour of $\left\langle R^{2}\right\rangle$, however, is deceptive. Although at first one might assume that for large $N$ the dependence of $\left\langle R^{2}\right\rangle$ on $N$ is linear, this is not the case! The relaxation to classical diffusion on the square percolation lattice has the form [23]

$$
\frac{\tilde{R}}{N^{1 / 2}}-\frac{\tilde{R}}{N^{1 / 2}}{ }_{N=\infty}=\frac{C}{N^{1 / 2}}
$$

for large $N$. Thus, if the results obtained are plotted in the form $\frac{\tilde{R}}{N^{1 / 2}}$ vs. $\frac{1}{N^{1 / 2}}$, the relaxation is evident from the tendency towards linearity as $\frac{1}{N^{1 / 2}} \rightarrow 0$. This is shown for $p=0.34$ in figure 2. The value of the classical diffusion coefficient $D$ is then the square of the intercept on the vertical axis.

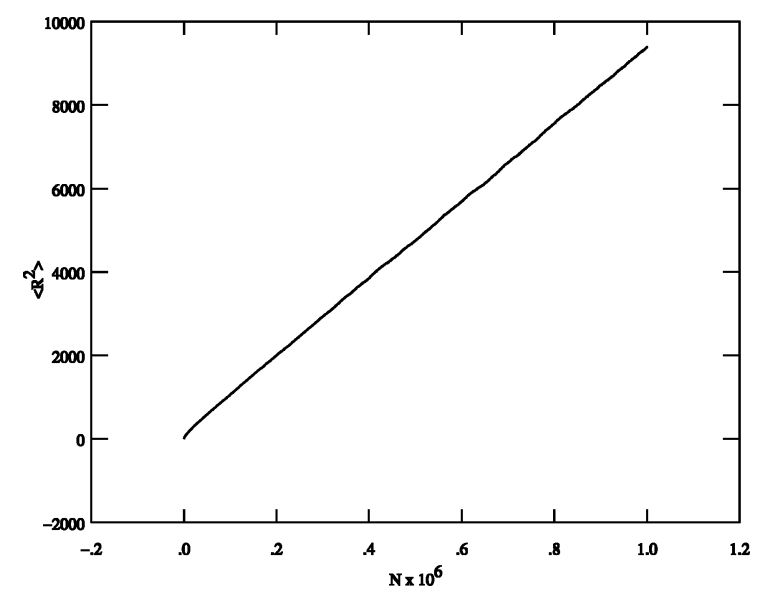

Fig. 1. The behaviour of $\left\langle R^{2}\right\rangle$ vs. $N$ for $p=0.34$.

A problem encountered in obtaining the intercept of graphs such as figure 2 is the presence of a small up- turn for large $N$ thought to be due to the effect of finite lattice size. This upturn becomes more pronounced as $p$ increases and is caused by walks that are able to proceed for longer than would otherwise be the case on the infinite lattice; imposing periodic boundary conditions on the finite lattice has the effect of joining together opposite sides of large finite clusters. As the threshold is approached, the onset of linear behaviour in the graph of $\frac{\tilde{R}}{N^{1 / 2}}$ vs. $\frac{1}{N^{1 / 2}}$ is postponed. This means that the intercept of such graphs becomes more difficult to determine. Therefore, to determine $D$ from these results it was assumed that the behaviour of the curve on the infinite lattice would be convex for all $N$. Thus, the region of the curve having the steepest slope was used to determine the intercept of the vertical axis from which $D$ was determined. Justification of this assumption is found by the behaviour of $\frac{\tilde{R}}{N^{1 / 2}}$ vs. $\frac{1}{N^{1 / 2}}$ observed on finite $d=2$ lattices which had much larger edge lengths; such behaviour was uniformly convex.

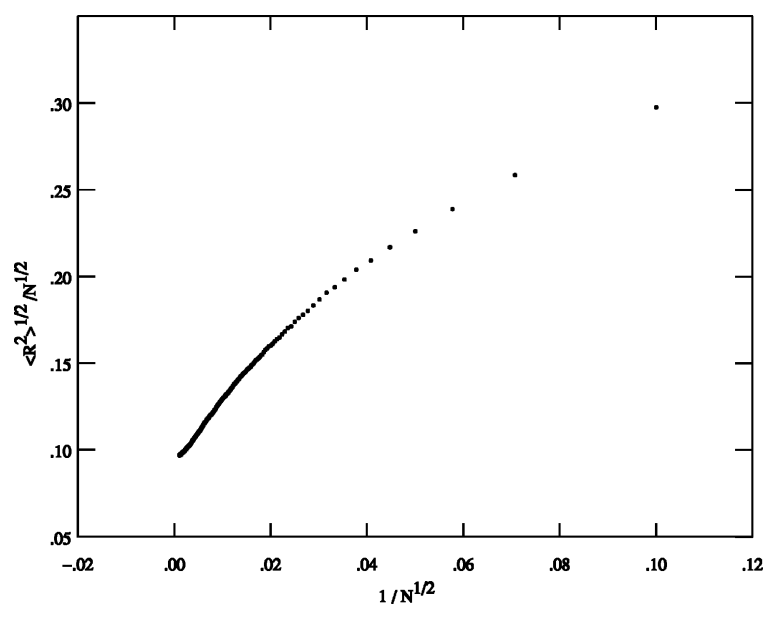

Fig. 2. The behaviour of $\frac{\tilde{R}}{N^{1 / 2}}$ vs. $\frac{1}{N^{1 / 2}}$ for $p=0.34$.

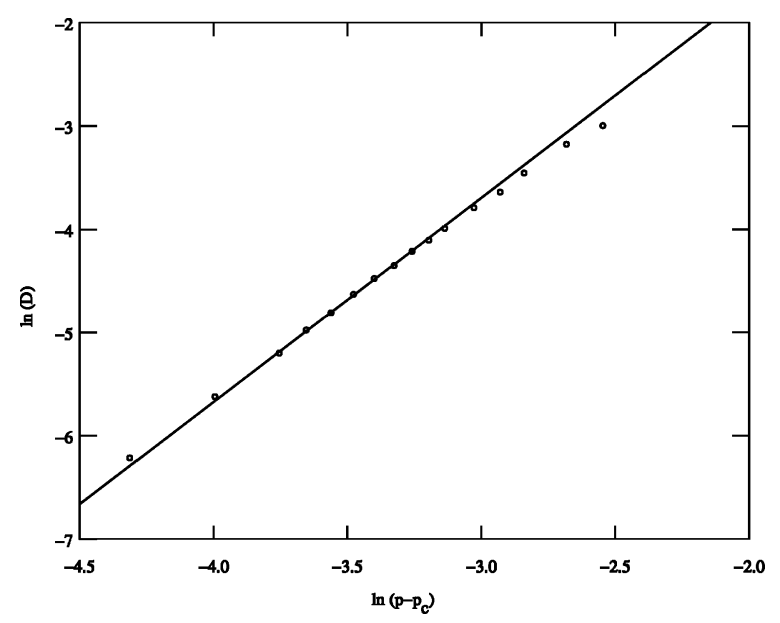

Fig. 3. The graph of $\ln (D)$ vs. $\ln \left(p-p_{c}\right)$. 
Recall equation (1). If scaling exists, then the graph of $\ln (D)$ vs. $\ln \left(p-p_{c}\right)$ will show linearity as the threshold is approached. This is shown for the results here in figure 3 where it is assumed that $p_{c}=0.3116$. As the threshold is approached, there is a clear region where the behaviour is linear. From the slope of the linear region of figure 3 the value of $\mu$ was found to be $\mu=1.980 \pm 0.025$. This is in good agreement with the most recent conductivity estimate for $d=3$ which gives $\mu=2.003 \pm 0.047$ and with the Alexander-Orbach conjecture. The extremely slow relaxation of percolation diffusion as the threshold is approached is clearly apparent by the behaviour of the leftmost point in the graph which fails to conform to the trend shown by the other points.

Evaluation of $\mu$ by the method given here can be compared to the simple approach of determining $D$ from the slope of the graph of $\left\langle R^{2}\right\rangle$ vs. $N$ for large $N$ as suggested by the crossover conjecture of Gefen et al [27]. Such an analysis of the results here gave $\mu \cong 1.81$, not much better than previously published work [22].

Given that the behaviour of percolation diffusion is characterised by a slow relaxation, one may wonder whether the relaxation itself has any scaling dependence on $\left(p-p_{c}\right)$. The rate of relaxation for diffusion is given by the constant, $C$, in equation (3). The constant $C$, which shall be termed the relaxation coefficient, was evaluated for different probabilities $p$ using the results here. It was assumed that a scaling law for $C$, similar to the other scaling laws discussed above, exists,

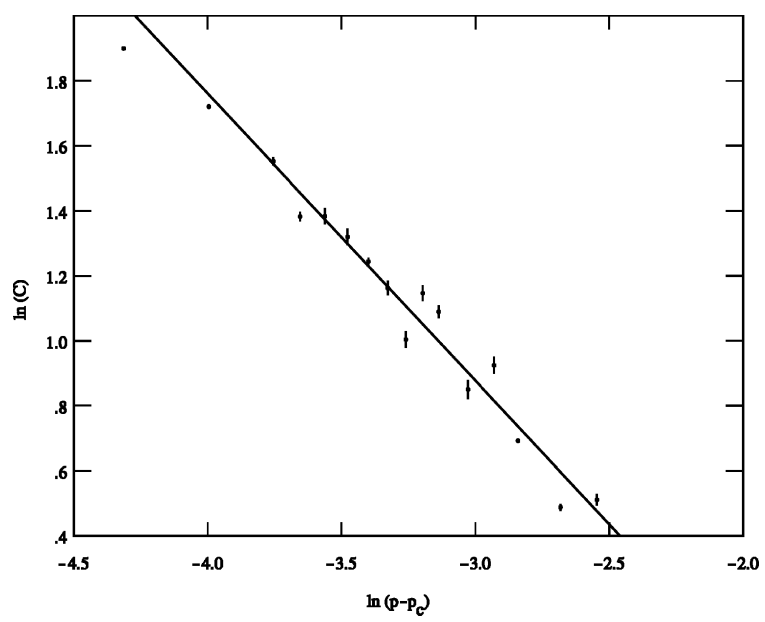

Fig. 4. The graph of $\ln (C)$ vs. $\ln \left(p-p_{c}\right)$.

$$
C \propto\left(p-p_{c}\right)^{\rho}
$$

and the graph of $\ln (C)$ vs. $\ln \left(p-p_{c}\right)$ was plotted as shown in figure 4 . The presence of a linear trend as the threshold is approached clearly implies scaling behaviour. From the slope of figure 4 , the value of $\rho$ was found to be $\rho=0.884 \pm 0.056$.

The value of $\rho$ given here is extremely close to the $d=3$ value of the exponent $\nu=0.88 \ldots$. A similar analysis [30] of the scaling behaviour of $C$ for the square lattice, however, gave $\rho \cong 1.68$. This is considerably larger than the $d=2$ value of $\nu=4 / 3$. The discrepancy may be understood by circumstantial evidence which points to a lower critical dimension for dynamic scaling, $d_{c}=3$, as discussed in [29]. For example, scaling relations such as the Alexander-Orbach conjecture appear to be supported by Monte Carlo work on $d=3$ lattices but not on $d=2$ lattices; certainly this has been shown to be the case here for $\mu$. The same may be true of the critical exponent for $C$, i.e., $\rho=\nu$ only when $d \geq 3$. At the moment this statement must remain a conjecture warranting further examination either by the analysis of scaling relations or by the simulation of diffusion on hyper-cubic percolation lattices.

\section{SUMMARY AND CONCLUSION}

The results presented here show that percolation diffusion on the cubic lattice undergoes a $\frac{1}{N^{1 / 2}}$ relaxation to asymptotic classical behaviour. Accounting for this relaxation enabled the expected scaling behaviour of $D$ to be observed. Thus, the dynamic exponent for diffusion on the cubic percolation lattice was found to be $\mu=$ $1.980 \pm 0.025$. It was shown that the relaxation coefficient, $C$, scaled with a critical exponent $\rho=0.884 \pm 0.056$, close to the $d=3$ value of the exponent $\nu=0.88 \ldots$. Future work on hyper-cubic lattices will show whether or not $d=3$ is a lower critical dimension for the identity $\rho=\nu$.

\section{ACKNOWLEDGEMENTS}

I acknowledge financial support from the School of Mathematical Studies, University of Portsmouth.
[1] S. R. Broadbent and J. M. Hammersly, Proc. Cambr. Phil. Soc. 53, 629 (1957).

[2] J. M. Hammersly, Annals of the Israeli Physical Society 5, 47 (1983).

[3] R. Zallen, Annals of the Israeli Physical Society 5, 3 (1983).

[4] D. Stauffer and A. Aharony, Introduction to Percolation Theory and its Applications, 2nd ed (Taylor and Fran- cis, 1991). This an excellent introduction to percolation theory with a strong emphasis on Monte Carlo work.

[5] A. Aharony, Directions in Condensed Matter Physics, ed. Mazenko and Grinsken (World Scientific, 1986).

[6] M. P. M. den Nijs, J. Phys. A 12, 1857 (1979).

[7] B. Nienhaus, J. Phys. A 15, 199 (1982).

[8] G. Grimmett, Percolation (Springer-Verlag, 1989).

[9] J. Adler, Y. Meir, A. Aharony, A. B. Harris and L. Klein, 
J. Stat. Phys, 58, No 3/4, 511 (1990); J. Adler, J. Phys. A 18, 307 (1984).

[10] H. Kesten, Ann. Inst. Henri. Poincare, 22, No 4, 425 (1986).

[11] K. Golden, Random Media and Composites, ed. by $R$. Kohn and G. Milton (SIAM, Philadelphia, 149, 1988).

[12] S. Kirkpatrick, Rev. Mod. Phys. 45, No 4, 574 (1973).

[13] J. P. Straley, Phys. Rev. B 15, No 12, 5733 (1977).

[14] Y. Yuge and K. Onizuka, J. Phys. C 11, L763 (1978).

[15] R. Fogelholm, J. Phys. C 13, L571 (1980).

[16] J. M. Normand, H. J. Herrman and M. Hajjar, J. Stat. Phys. 52, 441 (1988).

[17] D. B. Gingold and C. J. Lobb, Phys. Rev. B 42, No 13, 8220 (1990).

[18] J. Straley, J. Phys. C 13, 2991 (1980).

[19] R. B. Pandey, D. Stauffer, A. Margolina and J. G. Zabolitzky, J. Stat. Phys. 34, No 3/4, 427 (1984).
[20] J. G. Zabolitzky, Phys. Rev. B 30, No 7, 4077 (1984).

[21] C. D. Mitescu, H. Ottavi and J. Roussenq, AIP Conf. Proc. 40, 337 (1978).

[22] C. D. Mitescu and J Roussenq, Annals of the Israeli Physical Society 5, 81 (1983).

[23] O. J. Poole and D. W. Salt, J. Phys. A. 29, 7959 (1996).

[24] D. Stauffer, Phys. Rep. 54, No 1, 1 (1979) gives a review of scaling theory for percolation.

[25] P. G. De Gennes, La Recherche 72, No 7, 919 (1976).

[26] C. Mitescu and J Roussenq, C. R. Acad. Sci. Paris 283, 999 (1976).

[27] Y. Gefen, A. Aharony, and S. Alexander, Phys. Rev. Letts. 50, No 1, 77 (1983).

[28] S. Alexander and R. Orbach, J. Phys. Lett. (Paris) 43, L625 (1982).

[29] M. Sahimi, J. Phys. A, 17, L601 (1984).

[30] O. J. Poole, (1996), unpublished.

\title{
ДИФУЗІЯ НА НЕВПОРЯДКОВАНІЙ КУБІЧНІЙ ГРАТЩ НАД ПОРОГОМ ПРОТІКАННЯ: ДОСЛІДЖЕННЯ МЕТОДОМ МОНТЕ КАРЛО
}

\author{
О. Д. Пул \\ Школа математичних дослідженъ, Університет Портсмут, \\ Портсмут, P01 2EG, Англіл, е-mail: poole@sms.port.ac.uk
}

\begin{abstract}
Недавні дослідження процесу протікання на квадратній гратці усунули невідповідність, що існувала між перколяційною дифузією і провідністю пов'язаної з нею невпорядкованої сітки опорів. Основна увага у наших дослідженнях приділяється кубічній гратщі, для якої показано, що перколяційна дифузія і граткова провідність описуються тим самим набором критичних показників. Вперше приведено у відповідність поведінку дифузії та провідності на кубічній гратці. Отримані дані свідчать про те, що швидкість релаксації перколяційної дифузії має масштабну поведінку з показником, близьким за величиною до критичного показника граткової кореляційної довжини.
\end{abstract}

